ఠ

\title{
Establishing a detection method for CCNY: a potentially significant clinical investigative marker in NSCLC patients
}

This article was published in the following Dove Medical Press journal:

OncoTargets and Therapy

Li Ma

Meng Gu

Yu Teng

Weiying Li

Department of Cellular and Molecular Biology, Beijing Tuberculosis and Thoracic Tumor Research Institution/ Beijing Chest Hospital, Capital Medical University, Beijing I0II49, China
Correspondence: Weiying Li Department of Cellular and Molecular Biology, Beijing Tuberculosis and Thoracic Tumor Research Institution/ Beijing Chest Hospital, Capital Medical University, Courtyard 9 Beiguan Street,

Tongzhou, Beijing 101 I49, China

Tel +86 I82 01032168

Fax +86 01089509373

Email li_weiying4I2@aliyun.com
Background: CCNY, a novel cyclin family member, plays an increasingly important role in the progression of tumor invasion and metastasis, including lung cancer. However, the clinical significance of CCNY in non-small-cell lung cancer (NSCLC) patients is unknown.

Patients and methods: We prepared CCNY monoclonal antibodies, validated specific peptides by a peptide array, and established a double-antibody sandwich ELISA detection method. Then, we measured CCNY levels in 100 NSCLC patients and 50 healthy controls. A blinded validation was subsequently performed in 399 NSCLC patients and 150 healthy controls.

Results: We successfully prepared two specific mouse anti-human CCNY monoclonal antibodies and established a reliable and stable detection method. In the training set, serum CCNY was markedly increased in the NSCLC patients $(P<0.05)$ with an integrated area under the curve of 0.751 . With further analysis of the CCNY levels, there were no differences in age, sex, smoking status, tumor location, histologic subtype, or tumor size, but differences were observed in lymphatic $(P<0.001)$ and distant $(P<0.001)$ metastases in NSCLC patients. The CCNY[+] patients had a shorter survival time and progression-free survival than $\mathrm{CCNY}[-]$ patients at 3 -year follow-up $(P<0.001)$. The results were confirmed by the validation set.

Conclusion: Our study suggests that CCNY may be useful as a latent tumor marker to facilitate diagnosis and may be an effective indicator of tumor aggressiveness, playing an important role in the prognosis of NSCLC patients.

Keywords: non-small-cell lung cancer, CCNY, peptide array, diagnosis, prognosis, tumor marker

\section{Introduction}

Lung cancer is the leading cause of cancer-related death with secondary malignancies. Approximately $25 \%$ of all cancer-related mortality is due to lung cancer. ${ }^{1}$ The main pathological types of lung cancer are small-cell lung cancer accounting for $15 \%$ and non-small-cell lung cancer (NSCLC) accounting for $85 \%$ of the cases. ${ }^{2}$ Recent studies have shown that early-stage lung cancer diagnoses account for only $16 \%$, while the majority of lung cancers are diagnosed at an advanced stage. The 5-year relative survival rate for lung cancer is $18 \%$ after diagnosis. Based on the development of genetic sequencing technology and the discovery of immune inhibitors, personalized precision medicine has rapidly advanced, effectively improving the survival time and quality of life of lung cancer patients. However, early diagnosis is important to palliate symptoms and prolong patient survival time. The computed tomography scan is a routine examination tool, but it has low specificity and high false positives. ${ }^{3}$ Cytology and biopsy remains the gold standard for the diagnosis of lung cancer, and 
specimens are difficult to obtain. Noninvasive or minimally invasive inspection methods are an urgent clinical need for lung cancer.

The development of more accurate diagnostic and prognostic monitoring tools is the focus of clinical applications in lung cancer. New tumor-related biomarkers are being detected through continuous advancements in biotechnology, such as ctDNA, ${ }^{4,5}$ miRNA, ${ }^{6,7}$ circulating tumor cells, ${ }^{8,9}$ exosome proteins, ${ }^{10}$ etc. Serum proteins are a hot area of research for potential biomarkers of lung cancer with certain advantages such as mature detection methods, simple detection equipment, and easy clinical applications compared to other types of biomarkers. According to some studies, changes in the level of some serum biomarkers have been shown to precede the development of cancer disease symptoms. ${ }^{11-14}$

CCNY is the latest cell cycle protein to be identified from a human testis cDNA library. CCNY acts as a growth factor sensor to integrate extracellular signals within the cell cycle machinery. ${ }^{15-18}$ Recent studies have shown that CCNY may play an important role in cancer cell proliferation and metastasis, including breast cancer, ${ }^{19}$ ovarian cancer, ${ }^{20}$ lung cancer, ${ }^{21,22}$ colon cancer, ${ }^{23}$ glioma, ${ }^{24}$ etc. We previously found that CCNY mRNA was overexpressed in numerous lung cancer cell lines, ${ }^{21}$ and that the level of CCNY protein was significantly increased in lung cancer tissues. ${ }^{25}$ However, the precise biological function and clinical significance of CCNY remain unclear.

In this study, we first synthesized CCNY peptides as immunological antigens to immunize mice, prepared specific monoclonal antibodies, and verified the specificity of these antibodies using a peptide array chip. Second, we established a double-antibody sandwich ELISA detection method for serum CCNY. Finally, we examined the content of serum CCNY in different sets and evaluated the clinical significance of CCNY.

\section{Patients and methods}

\section{Serum and samples}

The training set serum samples from 100 patients with NSCLC together with 50 age-matched healthy controls were obtained from the Beijing Chest Hospital Samples Bank from May 2012 to May 2015. ${ }^{30}$ The validation set serum samples from 399 patients with NSCLC together with 150 age-matched healthy controls were also obtained from the Beijing Chest Hospital Samples Bank from July 2014 to July 2017. The clinical characteristics of the patients with NSCLC are shown in Table 1.
The patients' serum samples were collected at initial diagnosis. None of the patients had received preoperative chemotherapy or radiotherapy. The healthy controls were recruited during annual health examinations, and they showed no evidence of malignancy. Tumor histology was classified according to the WHO guidelines for histologic type of lung tumors. TNM stage was defined in accordance with the seventh edition of the TNM Classification of Lung Cancer. For survival analysis, the follow-up period was limited to 36 months. The study protocol was approved by the Beijing Chest Hospital Medical Ethics Committee according to the legal regulations.

The supernatants were obtained from the blood samples through centrifugation at $3,000 \times g$ for 15 minutes at $4^{\circ} \mathrm{C}$ and were immediately sub-packaged and stored at $-80^{\circ} \mathrm{C}$ for further analysis.

\section{Cell lines and mice}

Overexpression of the human Cyclin $Y$ gene in the NSCLC cell line H1299 was performed in our laboratory. Sp2/0 myeloma cells were purchased from the Institute of Basic Medical Sciences, Chinese Academy of Medical Sciences School of Basic Medical Sciences. BALB/c female mice were provided by Vital River, Beijing, China. All procedures were approved by the Capital Medical University Animal Care and Use Committee.

\section{Monoclonal antibody preparation of CCNY}

In the immune experiment, $0.1 \mathrm{mg}$ of the immune antigen peptide 1: C V S S S P K L R R N A H S R L E S Y R P D T D L S R E D T G and peptide 2: E N L H P L S K S E V P P D Y D K H N P E Q K Q I Y R F V R was synthesized and covalently coupled to keyhole limpet (KLH). To enhance peptide immunogenicity, the peptide antigen was blended with the same volume of Freund's complete adjuvant and injected into the backs of 18-20 g BALB/c female mice at 6 weeks of age by subcutaneous injection. Three weeks later, the same dose of the immune antigen was administered by intraperitoneal injection to induce the secondary immune response. Under sterile conditions, splenocytes were fused with $\mathrm{Sp} 2 / 0$ myeloma cells to make hybridoma cells according to a standard procedure. Conditioned media (HAT medium [hypoxanthine-aminopterin-thymidine selection medium]) from growing hybridoma cells were screened using the ELISA method. Select hybridoma clones produced antibodies that were highly specific for the respective target peptides. After limiting dilution of the hybridoma cells, two 
Table I Association between serum CCNY protein status and clinicopathological characteristics in NSCLC patients

\begin{tabular}{|c|c|c|c|c|c|c|}
\hline \multirow{2}{*}{$\begin{array}{l}\text { Group } \\
\text { Variables }\end{array}$} & \multicolumn{3}{|c|}{ Training set } & \multicolumn{3}{|c|}{ Validation set } \\
\hline & No & CCNY+ (\%) & $P$-value & No & CCNY+ (\%) & $P$-value \\
\hline \multicolumn{7}{|l|}{ Age (years) } \\
\hline$\leq 60$ & 217 & $70(32.3)$ & 0.196 & 56 & $32(57.1)$ & 0.107 \\
\hline$>60$ & 182 & $70(38.5)$ & & 44 & $18(40.9)$ & \\
\hline \multicolumn{7}{|l|}{ Gender } \\
\hline Male & 273 & I0I (37.0) & 0.240 & 72 & $34(47.2)$ & 0.373 \\
\hline Female & 126 & $39(31.0)$ & & 28 & $16(57.1)$ & \\
\hline \multicolumn{7}{|l|}{ Smoking history } \\
\hline Smoker & 240 & $88(36.7)$ & 0.417 & 63 & $32(50.8)$ & 0.836 \\
\hline Non-smoker & 159 & $52(32.7)$ & & 37 & $18(48.6)$ & \\
\hline \multicolumn{7}{|l|}{ Tumor location } \\
\hline Right & 165 & $58(35.2)$ & 0.982 & 48 & $28(58.3)$ & 0.109 \\
\hline Left & 234 & $82(35.0)$ & & 52 & $22(42.3)$ & \\
\hline \multicolumn{7}{|c|}{ Histologic subtype } \\
\hline $\mathrm{A}$ & 243 & $86(35.4)$ & 0.874 & 48 & $23(47.9)$ & 0.689 \\
\hline $\mathrm{S}$ & 156 & $54(34.6)$ & & 52 & $27(51.9)$ & \\
\hline \multicolumn{7}{|l|}{ pT status } \\
\hline PTI & 39 & $16(41.0)$ & 0.834 & 32 & $15(46.9)$ & 0.976 \\
\hline $\mathrm{pT} 2$ & 168 & $56(33.3)$ & & 43 & $22(5 \mid .2)$ & \\
\hline $\mathrm{pT3}$ & 55 & $19(34.5)$ & & 8 & $4(50)$ & \\
\hline pT4 & 137 & $49(35.8)$ & & 17 & $9(52.9)$ & \\
\hline \multicolumn{7}{|l|}{$\mathrm{pN}$ status } \\
\hline pNo & 87 & $13(14.9)$ & $<0.00 \mathrm{I}^{\mathrm{a}}$ & 30 & $9(30)$ & $0.022^{\mathrm{a}}$ \\
\hline $\mathrm{pNI}$ & 69 & $25(36.2)$ & & 24 & $13(54.2)$ & \\
\hline $\mathrm{pN} 2$ & 124 & 47 (37.9) & & 32 & $17(53.1)$ & \\
\hline $\mathrm{pN} 3$ & 119 & 55 (46.2) & & 14 & II (78.6) & \\
\hline \multicolumn{7}{|l|}{ pM status } \\
\hline pM0 & 220 & $63(28.6)$ & $0.003^{\mathrm{a}}$ & 65 & $25(38.5)$ & $0.002^{\mathrm{a}}$ \\
\hline $\mathrm{pMI}$ & 179 & $77(43.0)$ & & 35 & 25 (7I.4) & \\
\hline \multicolumn{7}{|l|}{ pTNM stage } \\
\hline $\mathrm{I}$ & 46 & $6(13.0)$ & 0.001 & 26 & $7(26.9)$ & 0.006 \\
\hline II & 65 & $17(26.2)$ & & 19 & $8(42.1)$ & \\
\hline III & 109 & $40(36.7)$ & & 20 & $10(50.0)$ & \\
\hline IV & 179 & $77(43)$ & & 35 & 25 (7I.4) & \\
\hline
\end{tabular}

Note: aStatistically significant at $P<0.05$.

Abbreviation: NSCLC, non-small-cell lung cancer.

clones produced monoclonal antibodies specific for CCNY peptide 1 and CCNY peptide 2 and were called MH001 and MH002, respectively. The mice were administered hybridoma cells producing MH001 or MH002 by intraperitoneal injection, and the abdomen of mice was observed until ascites formed. Cells were purified from the ascites, quantified, subpackaged, and stored at $-80^{\circ} \mathrm{C}$ for further analysis.

\section{CCNY peptide array chip}

Peptide arrays were prepared on amino-PEG500 cellulose membrane-UC540 (Intavis AG, Cologne, Germany) using a SPOT robot (Intavis AG) according to a standard spotsynthesis protocol. ${ }^{26}$ Special cellulose chromatography paper was chemically derived to carry spots of dipeptide anchors for the preparation of either immobilized peptide. Each peptide point was $10 \times 15 \mathrm{~cm}$ in size and contained 12 amino acid residues, overlapping three amino acids per point. CCNY is a 341 amino acid protein, so the CCNY peptide array contains 110 points (Figure S1). Twenty amino acids were dissolved in N-methylpyrrolidone at $0.05 \mathrm{mmol} / \mathrm{L}$. An Auto-Spot Robot generated a peptide array by a three-step procedure. First, an N-Fmoc-protected amino acid condenses peptide bond reactions, followed by Fmoc cleavage. Second, after each synthesis, all residual amino functions between the spots were blocked by acetylation with $2 \%$ acetic acid anhydride in dimethyl formamide. Finally, the peptides were then 
deprotected by a 1-hour treatment with dichloromethane and trifluoroacetic acid (1:1), containing 3\% tri-isopropylsilane and $2 \%$ water. The peptide array chip was used to validate the above-mentioned two antibodies specific for recognizing an immune antigen peptide.

\section{Based on peptide array chip, the specificity of the CCNY monoclonal antibody was validated}

Two peptide array chip membranes were blocked with 5\% w/v nonfat milk in PBS-T (PBS [pH 7.2] containing $0.1 \%$ v/v Tween-20) and incubated overnight at $4^{\circ} \mathrm{C}$ with $\mathrm{CCNY}$ monoclonal antibodies (MH001 and MH002) (1:100 dilution). Then, peroxidase-conjugated secondary antibodies (1:2,500 dilution, goat anti-mouse IgG, ab97040; Abcam) were incubated for 60 minutes at $37^{\circ} \mathrm{C}$. Finally, the immune reactive bands were visualized using an enhanced chemiluminescence kit (Thermo Fisher Scientific, Waltham, MA, USA) according to the manufacturer's instructions, and the images were acquired using an Alpha Innotech Digital Imaging System.

\section{Establishment of the CCNY protein detection method}

In total, $1 \mu \mathrm{g} / \mathrm{mL}$ of $\mathrm{MH001}$ and $\mathrm{MH} 002$, as envelope antibodies, was used to coat the 96-well microtiter plates. Pure CCNY protein was used as a standard sensing object, and goat anti-rabbit CCNY antibody (anti-CCNY antibody, ab80853; Abcam) was used as the detection antibody to validate the detection effect of the double-antibody sandwich ELISA detection method. The different concentration gradient of the standard CCNY protein was plotted on the X-axis, the absorbance on the $\mathrm{Y}$-axis, and a best fit curve was drawn through these points on the graph using CurveExpert 13.0.

\section{Detecting serum CCNY protein levels in different groups}

The 96-well microtiter plates were coated with $100 \mu \mathrm{L}$ per well of $1 \mu \mathrm{g} / \mathrm{mL}$ of envelope antibody and incubated overnight at $4{ }^{\circ} \mathrm{C}$. The wells were blocked with $5 \% \mathrm{w} / \mathrm{v}$ nonfat milk in PBS-T (PBS pH 7.2 containing $0.05 \% \mathrm{v} / \mathrm{v}$ Tween-20) for 60 minutes at $37^{\circ} \mathrm{C}$ and incubated for 90 minutes at $37^{\circ} \mathrm{C}$ with human serum samples diluted 1:10 in PBS. The wells were then washed five times with PBS-T and incubated with $0.1 \mu \mathrm{g} /$ $\mathrm{mL}$ detecting antibody for 60 minutes at $37^{\circ} \mathrm{C}$. Then, the wells were washed five times with PBS-T, followed by incubation with horseradish peroxidase-conjugated goat anti-rabbit IgG (ab6721; Abcam) diluted 1:5,000 for 60 minutes at $37^{\circ} \mathrm{C}$, and then washed again five times with PBS-T. Finally, $100 \mu \mathrm{L}$ of 3,3',5,5'-tetramethylbenzidine (TMB) was added to each well, followed by incubation for 15 minutes at room temperature. Subsequently, $50 \mu \mathrm{L}$ of $2 \mathrm{M} \mathrm{H}_{2} \mathrm{SO}_{4}$ was added to each well as a stop solution and mixed thoroughly. The OD at $450 \mathrm{~nm}$ was read using a spectrophotometer within 30 minutes.

\section{Quality control}

To ensure that the immunoassay was suitable for measuring clinical serum samples, reproducibility and linearity were examined. The assay showed excellent linearity with serial dilutions, intra-assay or inter-assay. We tested at least 25 times using the same sample and ensured that the coefficient of variation $(\mathrm{CV})$ for the intra-assay and inter-assay variability studies was $<10 \%$. Additionally, if samples fell outside of the linear detection range of the standard curves, the sample was rediluted and remeasured.

\section{Sample size determination}

$\mathrm{CCNY}$ is a new biomarker in lung cancer research. The expression level of CCNY in serum was not clear, and therefore a formal sample size calculation was not possible. Based on previous experiences, the positive rate range of most biomarkers was $25 \%-50 \%$ in a healthy control group, so we selected $>90 \%$ of normal control as the cutoff value. According to the comparison formula of two independent sample rates to calculate the minimum effective sample size, a significance level of $5 \%$ and power of $90 \%$ were used $(\alpha=0.05, \beta=0.1), \mathrm{N}=2 \times(\mathrm{u} \alpha+\mathrm{u} \beta) 2 \times \mathrm{P}(1-\mathrm{P}) /(\mathrm{P} 1-\mathrm{P} 2) 2$, $\mathrm{P}=(\mathrm{P} 1+\mathrm{P} 2) / 2$, where $\mathrm{P} 1$ and $\mathrm{P} 2$ are the $\mathrm{CCNY}$ positive rates in the NSCLC patient group and the healthy control group, respectively. The sample size was from 28 to 135 .

\section{Statistical analyses}

The data were analyzed using the SPSS 16.0 software package. Differences in the serum CCNY protein concentrations between the healthy control and the NSCLC patient groups were summarized as the mean and SD. A Student's $t$-test was used to evaluate the differences between the groups. Receiver operating characteristic (ROC) curves were drawn, and data $>90 \%$ of normal control were selected as the cutoff value. The chi-squared test was used to assess the independence of frequency counts in various groups. Progression-free survival (PFS) or overall survival (OS) was defined as the time interval between the date of diagnosis and the date of death or the last follow-up. Survival curves were calculated using the Kaplan-Meier method, and comparisons between the different groups were analyzed 
using the log-rank test. $P<0.05$ indicated a statistically significant difference.

\section{Results}

\section{The monoclonal antibodies specifically recognized the corresponding epitope of the antigen in the peptide array chip}

We successfully prepared two specific mouse anti-human CCNY monoclonal antibodies. Then, we used a peptide array chip to validate the specificity of these antibodies. The results showed that MH001 only specifically recognized the 10-40 aa site of CCNY peptide 1 as an immune antigen, and the cellulose nitrate member did not have a nonspecific binding site. Additionally, MH002 only specifically recognized the 149-178 aa site of CCNY peptide 2, and the results were also validated by pure CCNY protein and CCNY overexpressed H1299 cells by Western blot analysis (Figure 1).

\section{Detection methods for CCNY protein}

We used MH001 and MH002 as envelope antibodies and a goat anti-rabbit CCNY antibody as the detection antibody and constructed a double-antibody sandwich ELISA method. The results showed that successful matches were only observed between the MH002 and the goat anti-rabbit CCNY antibodies. In a follow-up experiment, a checkerboard synergy study was performed to determine the best working concentration of these two antibodies. We selected $1 \mu \mathrm{g} / \mathrm{mL}$ as the concentration of the envelope antibody and $0.4 \mu \mathrm{g} / \mathrm{mL}$ as the concentration of the detection antibody. The detection range of serum CCNY protein was from $0.0156 \mathrm{ng} / \mathrm{mL}$ to $1 \mathrm{ng} / \mathrm{mL}$. The CCNY protein standard curve was linear and had a good fitting degree of curvilinear regression. The $R^{2}$ of the standard curve was 0.9914 (Figure S2). Through multiple detection repetitions, the intra-CV of the detection methods for $\mathrm{CCNY}$ protein was calculated as $7.77 \%$ and the interCV was $6.28 \%$ (Table S1). The double-antibody sandwich ELISA method had good reproducibility and linearity and could guarantee the detection quality.

\section{The levels of CCNY protein were different between NSCLC patients and healthy controls}

In the training set, the serum CCNY level was markedly increased in 100 NSCLC patients compared with 50 healthy controls (NSCLC:Healthy, mean \pm SD , $0.894 \pm 0.433$ ng: $0.558 \pm 0.161 \mathrm{ng}$, $P<0.05)$. The ROC curve for the CCNY level had a sensitivity of $50 \%$ and a specificity of $90 \%$ at a cutoff value of $0.775 \mathrm{ng} / \mathrm{mL}$ and area under the curve (AUC) of 0.751 (Figure 2A and B). There were no differences in CCNY levels based on age, sex, smoking status, tumor location, histologic subtype, or tumor size in NSCLC patients. However, our results suggested that the serum CCNY level reflects the aggressive progression of both lymphatic $(P<0.001)$ and distant $(P<0.001)$ metastases in NSCLC (Table 1).

In the blind validation set, the result was similar to that obtained in the training set. The serum CCNY level was markedly increased in 399 NSCLC patients compared with 150 healthy controls (NSCLC:Healthy, mean $\pm \mathrm{SD}, 0.989 \pm 0.542 \mathrm{ng}$ : $0.576 \pm 0.355 \mathrm{ng}, P<0.05$ ). The ROC curve for the CCNY level had a sensitivity of $34.6 \%$ and a specificity of $90 \%$ at a cutoff value of $0.933 \mathrm{ng} / \mathrm{mL}$ and $\mathrm{AUC}=0.718$ (Figure 2C and D). The clinical pathological characteristic results of the

A
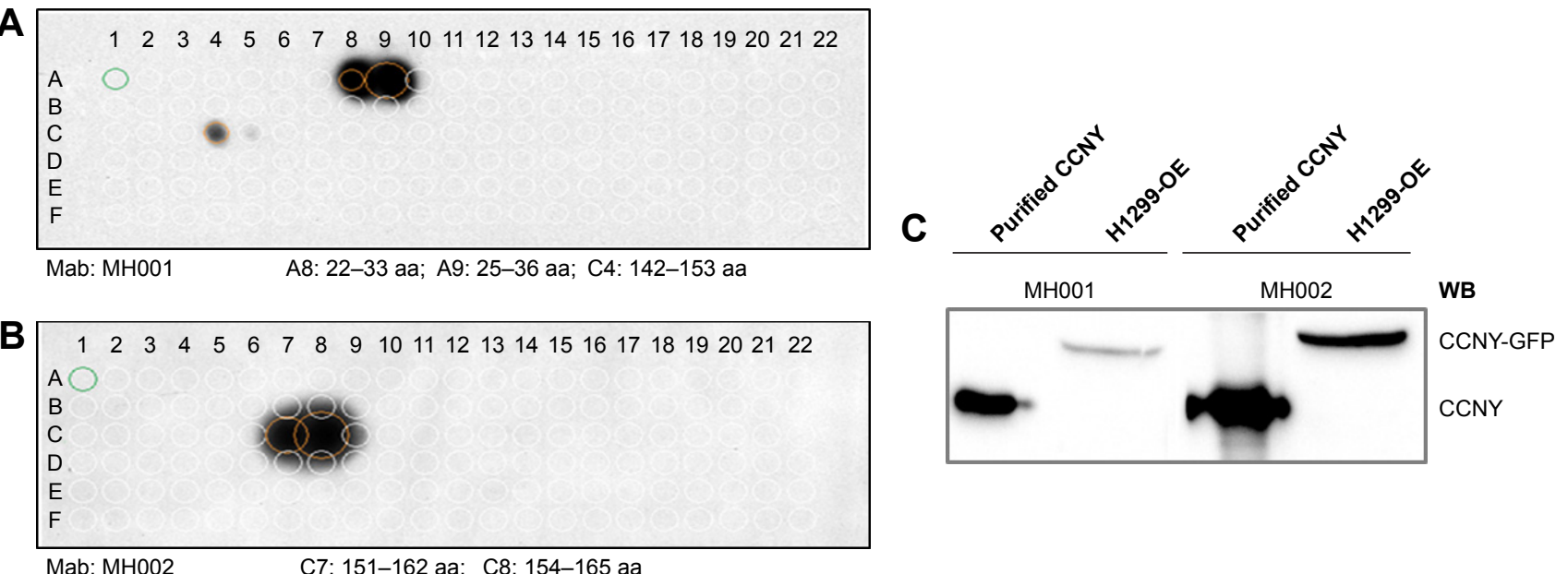

Figure I The specificity of $\mathrm{MHOOI}$ and $\mathrm{MH} 002$.

Notes: (A) MH00I specifically recognized peptide I (I0-40 aa). (B) MH002 specifically recognized peptide 2 (I49-I78 aa). (C) MH00I and MH002 recognized pure CCNY protein and CCNY overexpressed cell line HI299 by Western blot (WB) analysis. 

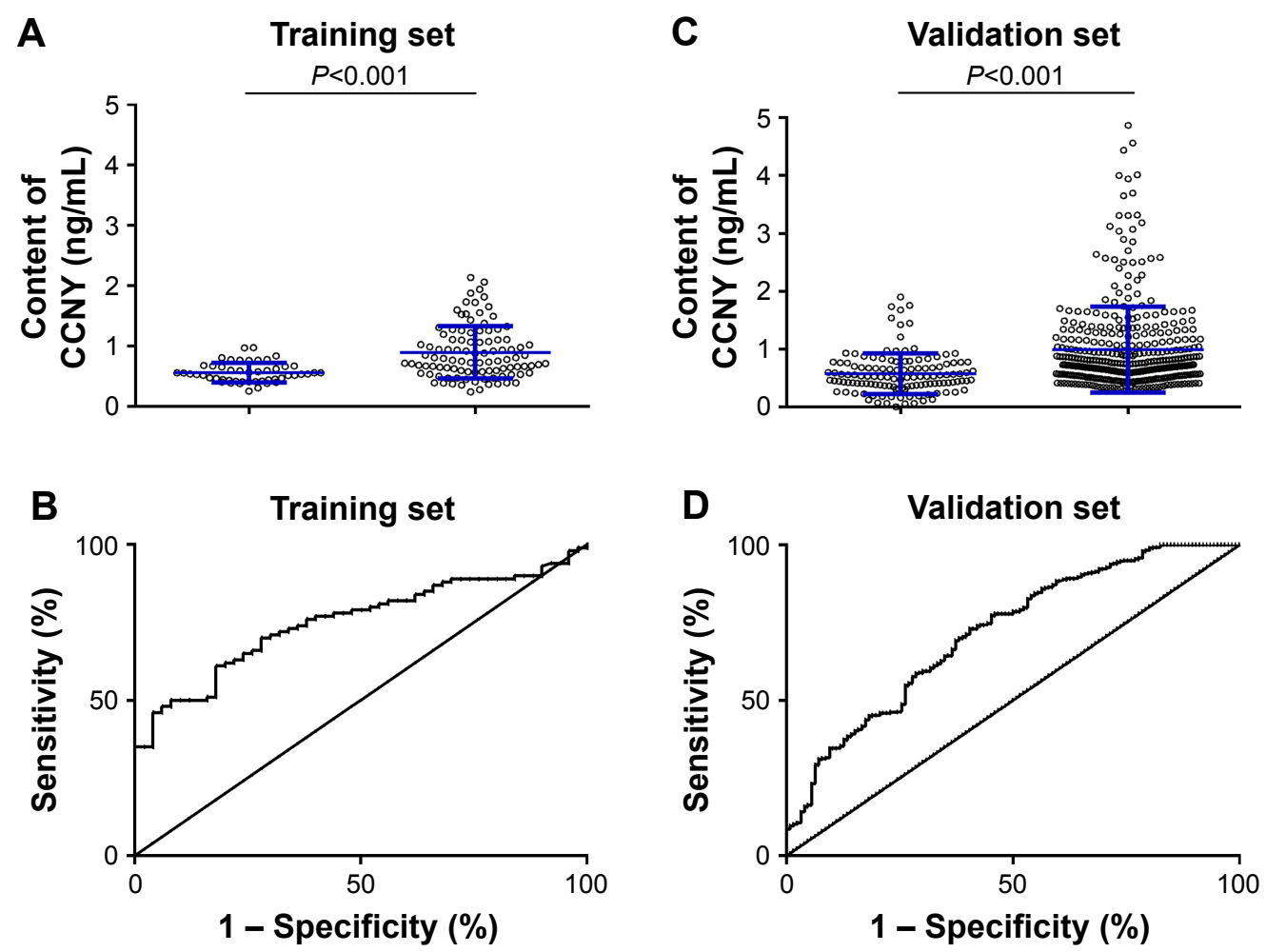

Figure 2 The concentration of CCNY protein in lung cancer patients and healthy controls.

Note: (A) Data distribution of CCNY in the training set, (B) ROC curve of the training set, (C) data distribution of CCNY in the validation set, and (D) ROC curve of the validation set.

Abbreviation: ROC, receiver operating characteristic.

blinded validation set were consistent with the results of the training set. There were no differences according to age, sex, smoking status, tumor location, histologic subtype, or tumor size in NSCLC patients, but the serum CCNY level reflects the aggressive progression of both lymphatic $(P<0.001)$ and distant $(P<0.001)$ metastases in NSCLC (Table 1).

We calculated the minimal sample size used to obtain the CCNY positive rate in both the training set and the validation set. The minimal sample size of the training set was 28 , and the minimal sample size of the validation set was 61 . In our study, the number of subjects far exceeded the minimal effective sample size and conformed to the requirements of the methodology.

\section{A significant relationship exists between CCNY and disease progression of NSCLC}

In our study, we further analyzed the relationship between the CCNY positive rate and TNM classification and clinical stage. In the training set, the results showed a significant relationship between CCNY positive rate and metastasis in NSCLC patients (M0:M1, 28.6\%:43\%, $P=0.003$ ). The validation set once again confirmed this result (M0:M1,
$38.5 \%: 71.4 \%, P=0.002$, Figure $3 \mathrm{~A}$ and $\mathrm{D})$. With the increase in N stage, the CCNY positive rate also increased in both the training set and the validation set (Figure 3B and E). Lastly, a comprehensive comparison between the CCNY positive rate and different clinical stages was performed. The CCNY positive rate in stage III and stage IV was significantly higher than that in stage I and stage II, especially stage I (Figure 3C and F). Therefore, we believe that there is a significant relationship between CCNY and disease progression of NSCLC.

\section{Significant difference in OS observed between $\mathrm{CCNY}[+]$ group and CCNY[-] group}

In the training set, all 100 patients were included in the following survival analysis. The mean survival time was 26.5 months (95\% CI 24.3-28.7 months), and the 3-year survival rate was $52.0 \%$. The levels of CCNY protein were evaluated for a possible association with OS. The KaplanMeier analysis showed a significant association between the levels of CCNY protein and OS for 3-year OS: CCNY protein positive group $(32.7 \%)$ vs CCNY protein negative group (72.9\%), and for mean OS and 95\% CI: CCNY protein positive group (21.3 months, 18.1-24.6 months) vs CCNY 

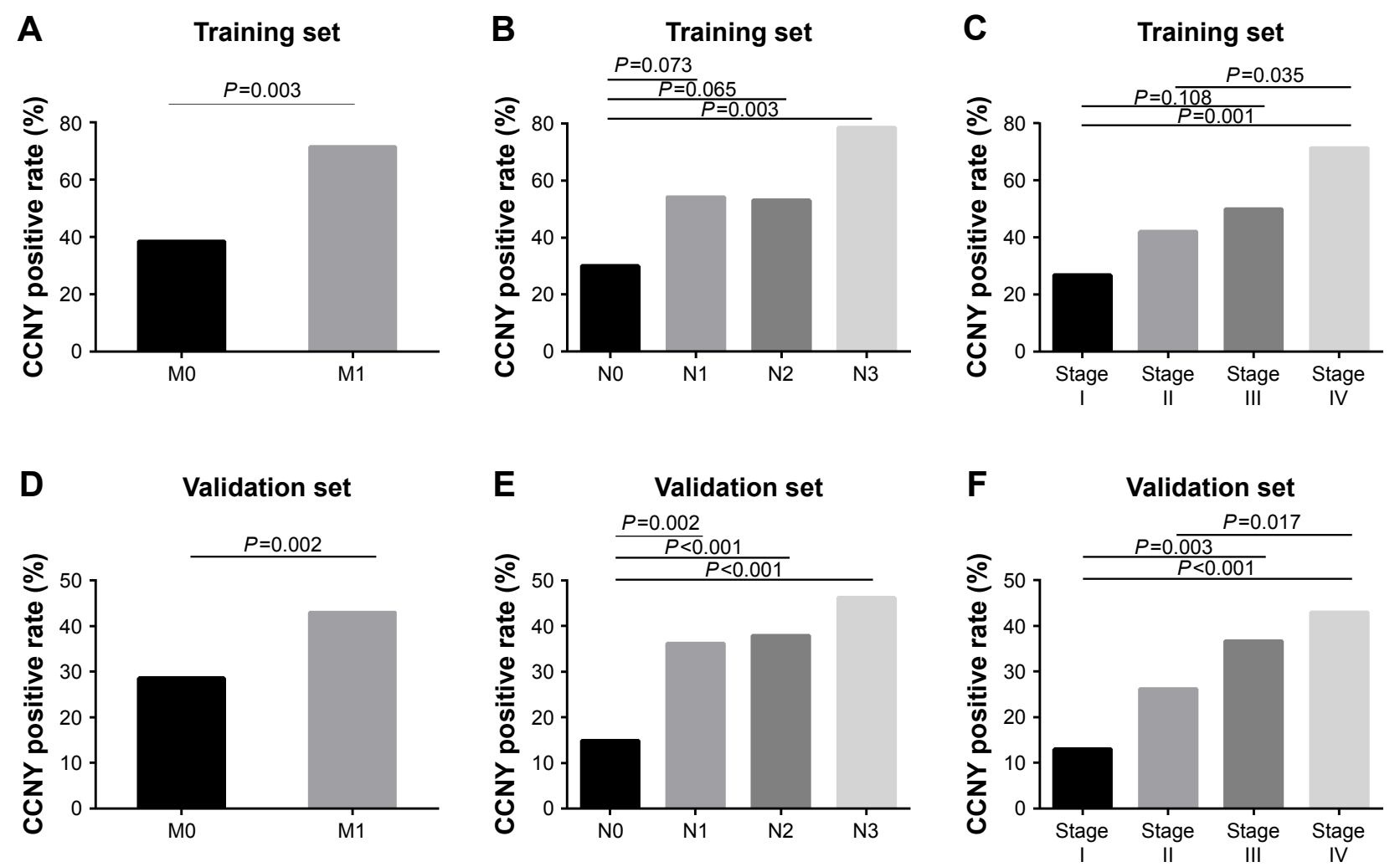

Figure 3 Variation in the CCNY positive rate according to the N, M, and clinical stage in NSCLC patients.

Note: In the training set, (A) positivity rates across consecutive $M$ stages in NSCLC patients, (B) positivity rates across consecutive N stages in NSCLC patients, and (C) positivity rates across consecutive clinical stages in NSCLC patients. In the validation set, (D) positivity rates across consecutive M stages in NSCLC patients, (E) positivity rates across consecutive N stages in NSCLC patients, and (F) positivity rates across consecutive clinical stages in NSCLC patients.

Abbreviation: NSCLC, non-small-cell lung cancer.

protein negative group (32.2 months, 30.1-34.2 months, $P<0.001)$. In the validation set, the prognosis results of NSCLC patients were consistent with the training set, and 318 patients were included in the following survival analysis. The median survival time was 23.3 months $(95 \%$ CI 22.1-24.5 months), and the 3-year survival rate was $37.4 \%$. The Kaplan-Meier analysis showed a significant association between the levels of CCNY protein and OS for 3-year OS: CCNY protein positive group (27.7\%) vs CCNY protein negative group (44.1\%), and for mean OS and $95 \%$ CI: CCNY protein positive group (19.2 months, 17.3-21.2 months) vs CCNY protein negative group (26.0 months, 24.6-27.4 months, $P<0.001)$. In summary, serum CCNY protein may be a valuable marker to evaluate survival time in NSCLC patients (Figure 4A and C).

\section{Serum CCNY protein may be a good prognosis monitoring marker in NSCLC patients}

To estimate the influence of differential disease progression on the living state of NSCLC patients, we also analyzed the relationship between the levels of CCNY protein and PFS. The results showed that, in the training set, the CCNY protein positive group had a PFS rate of $30.0 \%$ vs $62.0 \%$ in the CCNY protein negative group, and the mean PFS and 95\% CI were 20.7 months and 17.2-24.2 months for the CCNY protein positive group vs 31.7 months and 29.4-34.1 months for the CCNY protein negative group $(P<0.001)$. In the validation set, the PFS rate of the CCNY protein positive group was $20.0 \%$ vs $36.2 \%$ for the CCNY protein negative group (mean PFS and 95\% CI: CCNY protein positive group [14.5 months, 12.5-16.4 months] vs CCNY protein negative group [20.3 months, 18.3-22.2 months], $P<0.001$ ). The serum CCNY protein changed before the tumor changed, so CCNY may be a good prognosis monitoring marker in NSCLC patients (Figure 4B and D).

\section{Discussion}

Presently, numerous detection methods are used in cancer patients. Biomarker detection is one of the most common detection methods in the clinic. Blood samples are relatively easy to obtain and tumor-associated proteins are frequently 

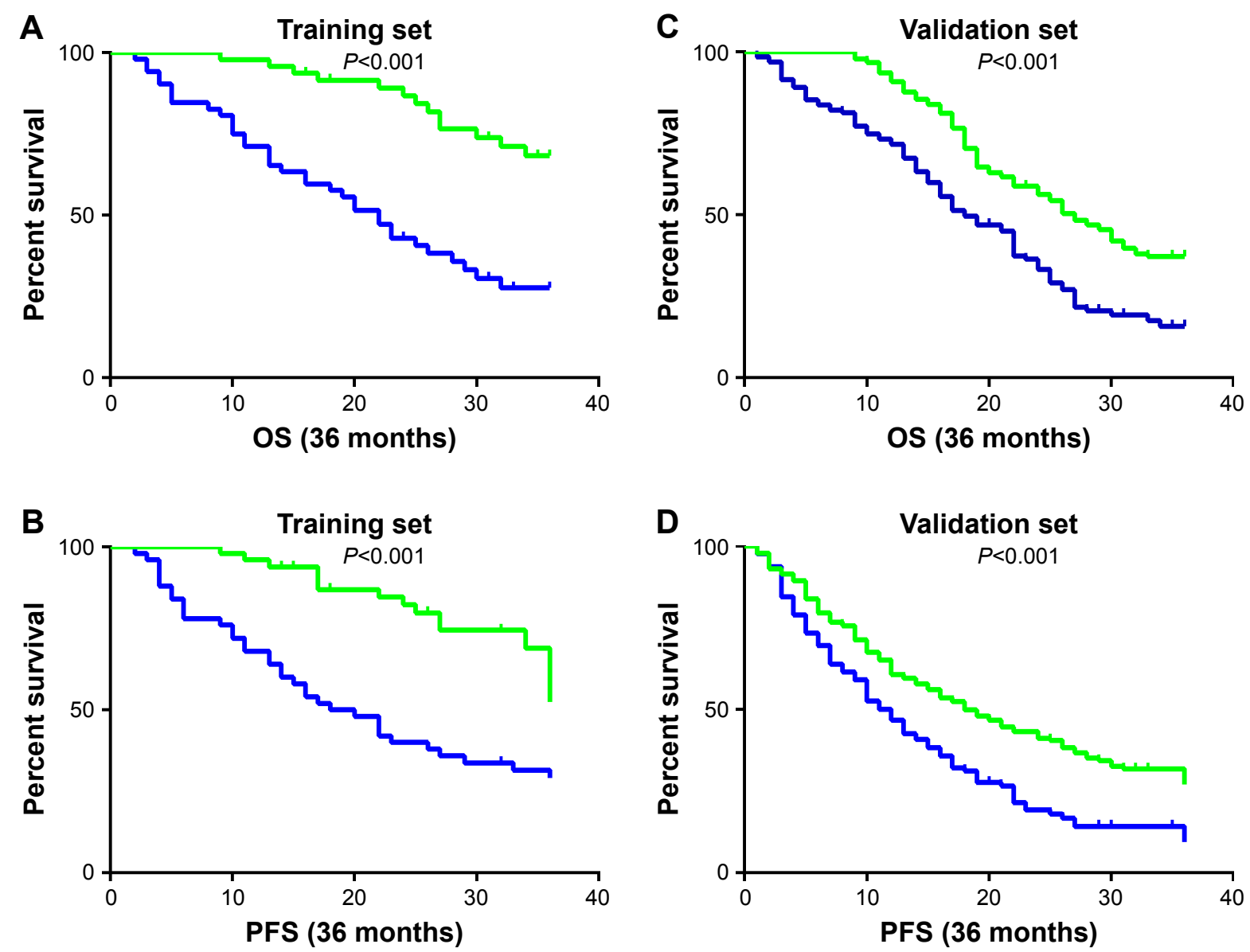

Figure 4 Kaplan-Meier analyses of overall survival (OS) and progression-free survival (PFS) in all eligible cases.

Notes: In the training set, $(\mathbf{A})$ the 3 -year OS rate was $32.7 \%$ for CCNY-positive $(C C N Y[+])$ patients and $72.9 \%$ for $C C N Y$-negative $(C C N Y[-])$ patients $(P<0.01)$. $(\mathbf{B})$ The 3-year PFS rate was $30 \%$ for CCNY[+] patients and $62 \%$ for CCNY[-] patients $(P<0.01)$. In the validation set, $(\mathbf{C})$ the 3 -year OS rate was $27.7 \%$ for $C C N Y[+]$ patients and $44.1 \%$ for CCNY[-] patients $(P<0.01)$. (D) The 3-year PFS rate was $20 \%$ for CCNY $[+]$ patients and $36.2 \%$ for CCNY[-] patients $(P<0.01)$. The blue line is CCNY-positive patients and the green line is CCNY-negative patients.

found in the serum of cancer patients. ${ }^{14,27-29}$ As the direct performer of physiological and pathological processes, protein expression profiles change in different diseases, even in different stages of the same disease, making protein level changes the basis of all detection technologies. If changes in protein levels in the blood can predict malignant changes of disease in patients, then we can detect these protein changes to predict disease progression. This type of study has been previously reported in the literature. ${ }^{30-33}$ Therefore, the aim of this experiment was to establish a means of detection based on blood samples to measure changes in CCNY protein.

Recent studies have shown that CCNY protein may play an important role in cancer cell proliferation and metastasis..$^{21,22}$ Our previous study have shown that CCNY protein is overexpressed in NSCLC tissues. ${ }^{25}$ Due to the lack of a detection method, we first successfully prepared CCNY monoclonal antibodies (MH001 and MH002) and validated the specificity of these monoclonal antibodies via peptide array chip to ensure the credibility and accuracy of the result. Next, the double-antibody sandwich ELISA method was established. To ensure increased detection accuracy and repeatability, we established stringent quality control standards. Finally, in this experiment, we designed two groups to detect the serum CCNY protein levels: the training set and the validation set. The small sample of the training set was used to discuss the potential clinical value of CCNY protein in the NSCLC patient group and the healthy group, and the large sample of the validation set was used to repeat and validate the experiments of the training set, further enhancing the reliability of these results. The detection result showed that the serum CCNY level was markedly increased in NSCLC patients compared with healthy controls, with increasing sample size. Regarding specificity consistency, the sensitivity declined slightly, but the AUC was $>0.7$. Low sensitivity is a limitation for single biomarker detection, so the CCNY protein has only subsidiary value in diagnosing 
NSCLC. The results also showed that the level of CCNY protein was correlated to the aggressive progression of both lymphatic $(P<0.001)$ and distant $(P<0.001)$ metastases in both the training set and the validation set. Especially, with the increase in $\mathrm{N}$ stage, $\mathrm{M}$ stage, and clinical stage, the positive rate of CCNY increased as well. The results suggested that CCNY protein may change with disease progression. Therefore, dynamically monitoring CCNY level changes to predict disease progression may provide a reference for the clinical treatment of patients with lung cancer. There were significant differences in the OS and PFS in the $\mathrm{CCNY}[+]$ group and $\mathrm{CCNY}[-]$ group. $\mathrm{CCNY}[+]$ patients were associated with poor prognosis, and the results suggested that serum CCNY protein may be a good prognosis monitoring marker for NSCLC patients in the future.

\section{Conclusion}

In summary, our data indicated that the serum CCNY level was significantly increased in the serum of NSCLC patients and may be useful as a latent tumor marker to facilitate NSCLC diagnosis. Additionally, serum CCNY may be an effective indicator of tumor aggressiveness and play an important role in the prognosis of NSCLC patients.

\section{Acknowledgment}

This study was supported by Beijing Municipal Administration of Hospitals Incubating Program, code PX2016062, awarded to Li Ma.

\section{Disclosure}

The authors report no conflicts of interest in this work.

\section{References}

1. Siegel RL, Miller KD, Jemal A. Cancer statistics, 2018. CA Cancer J Clin. 2018;68(1):7-30.

2. Oser MG, Niederst MJ, Sequist LV, Engelman JA. Transformation from non-small-cell lung cancer to small-cell lung cancer: molecular drivers and cells of origin. Lancet Oncol. 2015;16(4):e165-e172.

3. Macmahon H, Austin JH, Gamsu G, et al. Guidelines for management of small pulmonary nodules detected on CT scans: a statement from the Fleischner Society. Radiology. 2005;237(2):395-400.

4. Lee Y, Park S, Kim WS, et al. Correlation between progression-free survival, tumor burden, and circulating tumor DNA in the initial diagnosis of advanced-stage EGFR-mutated non-small cell lung cancer. Thorac Cancer. 2018;9(9):1104-1110.

5. Yang X, Zhuo M, Ye X, et al. Quantification of mutant alleles in circulating tumor DNA can predict survival in lung cancer. Oncotarget. 2016; 7(15):20810-20824.

6. Wu Y, Zhang J, Hong Y, Wang X. Effects of Kanglaite injection on serum miRNA-21 in patients with advanced lung cancer. Med Sci Monit. 2018;24:2901-2906.
7. Liu Q, Yu Z, Yuan S, et al. Circulating exosomal microRNAs as prognostic biomarkers for non-small-cell lung cancer. Oncotarget. 2017; 8(8):13048-13058.

8. Kapeleris J, Kulasinghe A, Warkiani ME, et al. The prognostic role of circulating tumor cells (CTCs) in lung cancer. Front Oncol. 2018; $8: 311$.

9. Watanabe M, Kenmotsu $\mathrm{H}$, Ko R, et al. Isolation and molecular analysis of circulating tumor cells from lung cancer patients using a microfluidic CHIP type cell sorter. Cancer Sci. 2018;109(8):2539-2548.

10. Sandfeld-Paulsen B, Jakobsen KR, Bæk R, et al. Exosomal proteins as diagnostic biomarkers in lung cancer. $J$ Thorac Oncol. 2016;11(10): 1701-1710.

11. Chapman CJ, Thorpe AJ, Murray A, et al. Immunobiomarkers in small cell lung cancer: potential early cancer signals. Clin Cancer Res. 2011;17(6):1474-1480.

12. Bracci PM, Zhou M, Young S, Wiemels J. Serum autoantibodies to pancreatic cancer antigens as biomarkers of pancreatic cancer in a San Francisco Bay area case-control study. Cancer. 2012;118(21): 5384-5394.

13. Lu H, Goodell V, Disis ML. Humoral immunity directed against tumorassociated antigens as potential biomarkers for the early diagnosis of cancer. J Proteome Res. 2008;7(4):1388-1394.

14. Vykoukal J, Sun N, Aguilar-Bonavides C, et al. Plasma-derived extracellular vesicle proteins as a source of biomarkers for lung adenocarcinoma. Oncotarget. 2017;8(56):95466-95480.

15. Satyanarayana A, Kaldis P. Mammalian cell-cycle regulation: several Cdks, numerous cyclins and diverse compensatory mechanisms. Oncogene. 2009;28(33):2925-2939.

16. Santamaria D, Ortega S. Cyclins and CDKs in development and cancer: lessons from genetically modified mice. Front Biosci. 2006;11: $1164-1188$.

17. Galderisi U, Jori FP, Giordano A. Cell cycle regulation and neural differentiation. Oncogene. 2003;22(33):5208-5219.

18. Jin J, Smith FD, Stark C, et al. Proteomic, functional, and domain-based analysis of in vivo 14-3-3 binding proteins involved in cytoskeletal regulation and cellular organization. Curr Biol. 2004;14(16):1436-1450.

19. Yan F, Wang X, Zhu M, Hu X. RNAi-mediated downregulation of cyclin Y to attenuate human breast cancer cell growth. Oncol Rep. 2016;36(5):2793-2799.

20. Liu H, Shi H, Fan Q, Sun X. Cyclin Y regulates the proliferation, migration, and invasion of ovarian cancer cells via Wnt signaling pathway. Tumour Biol. 2016;37(8):10161-10175.

21. Yue W, Zhao X, Zhang L, et al. Overexpression of cyclin Y in non-small cell lung cancer is associated with cancer cell proliferation. Sci China Life Sci. 2010;53(4):511-516.

22. Yue $\mathrm{W}$, Zhao $\mathrm{X}$, Zhang $\mathrm{L}$, et al. Cell cycle protein cyclin $\mathrm{Y}$ is associated with human non-small-cell lung cancer proliferation and tumorigenesis. Clin Lung Cancer. 2011;12(1):43-50.

23. Ying-Tao Z, Yi-Ping G, Lu-Sheng S, Yi-Li W. Proteomic analysis of differentially expressed proteins between metastatic and non-metastatic human colorectal carcinoma cell lines. Eur J Gastroenterol Hepatol. 2005;17(7):725-732.

24. Xu Y, Wang Z, Wang J, Li J, Wang H, Yue W. Lentivirus-mediated knockdown of cyclin Y (CCNY) inhibits glioma cell proliferation. Oncol Res. 2010;18(8):359-364.

25. Ma L, Yue W, Teng Y, Zhang L, Gu M, Wang Y. Serum anti-CCNY autoantibody is an independent prognosis indicator for postoperative patients with early-stage nonsmall-cell lung carcinoma. Dis Markers. 2013;35(5):317-325.

26. Beckmann C, Haase B, Timmis KN, Tesar M. Multifunctional g3ppeptide tag for current phage display systems. J Immunol Methods. 1998;212(2):131-138.

27. Chapman CJ, Murray A, McElveen JE, et al. Autoantibodies in lung cancer: possibilities for early detection and subsequent cure. Thorax. 2008;63(3):228-233. 
28. Fernández-Grijalva AL, Aguilar-Lemarroy A, Jave-Suarez LF, et al. Alpha 2HS-glycoprotein, a tumor-associated antigen (TAA) detected in Mexican patients with early-stage breast cancer. J Proteomics. 2015;112:301-312.

29. Ummanni R, Duscharla D, Barett C, et al. Prostate cancer-associated autoantibodies in serum against tumor-associated antigens as potential new biomarkers. J Proteomics. 2015;119:218-229.

30. Avrillon V, Locatelli-Sanchez M, Folliet L, et al. Lung cancer may increase serum procalcitonin level. Infect Disord Drug Targets. 2015; 15(1):57-63.
31. Katono K, Sato Y, Kobayashi M, et al. Clinicopathological significance of S100A14 expression in lung adenocarcinoma. Oncol Res Treat. 2017;40(10):594-602.

32. Ayyub A, Saleem M, Musharraf SG, Naz M, Tariq A, Hashmi N. Mass spectrometric identification, characterization and validation of the haptoglobin $\beta$-chain protein as a lung cancer serum biomarker. $\mathrm{Mol}$ Med Rep. 2015;12(3):3755-3762.

33. Korkmaz ET, Koksal D, Aksu F, et al. Triple test with tumor markers CYFRA 21.1, HE4, and ProGRP might contribute to diagnosis and subtyping of lung cancer. Clin Biochem. 2018;58:15-19. 


\section{Supplementary materials}

\begin{tabular}{|c|c|c|c|}
\hline 1 pkmgnttsccvs & 30 nhhppglfinhh & 59 npeqkqiyrfvr & 88 lerqflellqfn \\
\hline 2 gnttsccvsssp & 31 ppglfinhhppg & 60 qkqiyrfvrtlf & 89 qflellqfninv \\
\hline 3 tsccvssspklr & 32 Ifinhhppgqia & 61 iyrfvrtlfsaa & 90 ellqfninvpss \\
\hline 4 cvssspkIrrna & 33 nhhppgqiarky & 62 fvrtlfsaaqlt & 91 qfninvpssvya \\
\hline 5 sspklrrnahsr & 34 ppgqiarkyssc & 63 tlfsaaqltaec & 92 invpssvyakyy \\
\hline 6 klrrnahsrles & 35 qiarkysscsti & 64 saaqltaecaiv & 93 pssvyakyyfdl \\
\hline 7 rnahsrlesyrp & 36 rkysscstifld & 65 qltaecaivtlv & 94 vyakyyfdlrs \\
\hline 8 hsrlesyrptdl & 37 sscstiflddst & 66 aecaivtlvyle & 95 kyyfdlrslaea \\
\hline 9 lesyrptdlsre & 38 stiflddstvsq & 67 aivtlvylerll & 96 fdlrslaeannl \\
\hline 10 yrptdlsredtg & 39 flddstvsqpnl & 68 tlvylerlltya & 97 rslaeannlsfp \\
\hline 11 tdlsredtgcnl & 40 dstvsqpnlkyt & 69 ylerlltyaeid & 98 aeannlsfplep \\
\hline 12 sredtgcnlqhi & 41 vsqpnlkytikc & 70 rlltyaeidicp & 99 nnlsfpleplsr \\
\hline 13 dtgcnlqhisdr & 42 pnlkytikcval & 71 tyaeidicpanw & 100 sfpleplsrera \\
\hline 14 cnlqhisdreni & 43 kytikcvalaiy & 72 eidicpanwkri & 101 leplsrerahkl \\
\hline 15 qhisdreniddl & 44 ikcvalaiyyhi & 73 icpanwkrivlg & 102 Isrerahkleai \\
\hline 16 sdreniddlnme & 45 valaiyyhiknr & 74 anwkrivlgail & 103 erahkleaisrl \\
\hline 17 eniddlnmefnp & 46 aiyyhiknrdpd & 75 krivlgaillas & 104 hkleaisrlced \\
\hline 18 ddlnmefnpsdh & 47 yhiknrdpdgrm & 76 vlgaillaskvw & 105 eaisrlcedkyk \\
\hline 19 nmefnpsdhpra & 48 knrdpdgrmlld & 77 aillaskvwddq & 106 srlcedkykdlr \\
\hline 20 fnpsdhprasti & 49 dpdgrmlldifd & 78 laskvwddqavw & 107 cedkykdlrrsa \\
\hline 21 sdhprastifls & 50 grmlldifdenl & 79 kvwddqavwnvd & 108 kykdlrrsarkr \\
\hline 22 prastiflsksq & 51 Ildifdenlhpl & 80 ddqavwnvdycq & 109 dlrrsarkrsas \\
\hline 23 stiflsksqtdv & 52 ifdenlhplsks & 81 avwnvdycqilk & 110 rsarkrsasadn \\
\hline 24 flsksqtdvrek & 53 enlhplsksevp & 82 nvdycqilkdit & 111 rkrsasadnltl \\
\hline 25 ksqtdvrekrks & 54 hplsksevppdy & 83 ycqilkditved & 112 sasadnlt|prw \\
\hline 26 tdvrekrkslfi & 55 sksevppdydkh & 84 ilkditvedmne & 113 adnltlprwspa \\
\hline 27 rekrkslfinhh & 56 evppdydkhnpe & 85 ditvedmneler & 114 Itlprwspaiis \\
\hline 28 rkslfinhhppg & 57 pdydkhnpeqkq & 86 vedmnelerqfl & 113 adnltlprwspa \\
\hline 29 Ifinhhppglfi & 58 dkhnpeqkqiyr & 87 mnelerqflell & 114 It|prwspaiis \\
\hline
\end{tabular}

Figure SI Amino acid sequence of each site in the peptide array chip.

A

\begin{tabular}{lll}
\hline No & $\begin{array}{l}\text { Standard } \\
\text { concentration }(\mathbf{n g} / \mathbf{m L})\end{array}$ & OD \\
\hline 1 & 0 (blank) & 0.061 \\
2 & 0.0156 & 0.103 \\
3 & 0.0313 & 0.145 \\
4 & 0.0625 & 0.232 \\
5 & 0.125 & 0.398 \\
6 & 0.25 & 0.717 \\
7 & 0.5 & 1.211 \\
8 & 1 & 2.058 \\
\hline
\end{tabular}

B

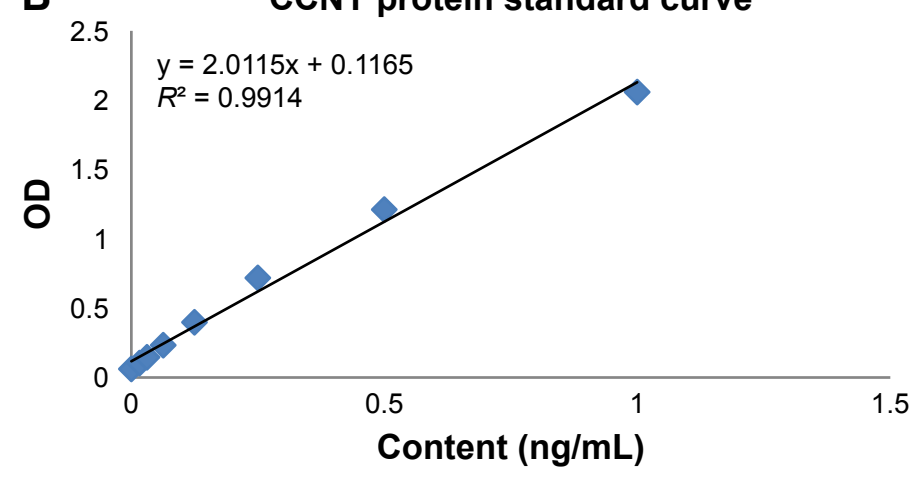

Figure S2 Standard curve of CCNY protein.

Notes: (A) standard curve of CCNY protein; (B) standard curve of CCNY protein. 
Table SI The detection of CV of CCNY protein

\begin{tabular}{|c|c|c|}
\hline Sample no & Inter-CV & Intra-CV \\
\hline I & 0.2340 & 0.2392 \\
\hline 2 & 0.2364 & 0.2423 \\
\hline 3 & 0.2497 & 0.2596 \\
\hline 4 & 0.2566 & 0.2685 \\
\hline 5 & 0.2610 & 0.2744 \\
\hline 6 & 0.2787 & 0.2973 \\
\hline 7 & 0.2794 & 0.2982 \\
\hline 8 & 0.2833 & 0.3032 \\
\hline 9 & 0.2833 & 0.3032 \\
\hline 10 & 0.2317 & 0.2362 \\
\hline II & 0.2293 & 0.2331 \\
\hline 12 & 0.2472 & 0.2563 \\
\hline 13 & 0.2463 & 0.2552 \\
\hline 14 & 0.2506 & 0.2608 \\
\hline 15 & 0.2675 & 0.2828 \\
\hline 16 & 0.2682 & 0.2837 \\
\hline 17 & 0.2719 & 0.2885 \\
\hline 18 & 0.2719 & 0.2885 \\
\hline 19 & 0.2548 & 0.2663 \\
\hline 20 & 0.2523 & 0.2629 \\
\hline 21 & 0.2719 & 0.2885 \\
\hline 22 & 0.2709 & 0.2872 \\
\hline 23 & 0.2757 & 0.2934 \\
\hline 24 & 0.2648 & 0.2793 \\
\hline 25 & 0.2655 & 0.2802 \\
\hline Mean & 0.2601 & 0.2731 \\
\hline SD & 0.01637 & 0.0212 \\
\hline CV (\%) & 6.28 & 7.77 \\
\hline
\end{tabular}

Abbreviation: $\mathrm{CV}$, coefficient of variation.

\section{Publish your work in this journal}

OncoTargets and Therapy is an international, peer-reviewed, open access journal focusing on the pathological basis of all cancers, potential targets for therapy and treatment protocols employed to improve the management of cancer patients. The journal also focuses on the impact of management programs and new therapeutic agents and protocols on

\section{Dovepress}

patient perspectives such as quality of life, adherence and satisfaction. The manuscript management system is completely online and includes a very quick and fair peer-review system, which is all easy to use. Visit http://www.dovepress.com/testimonials.php to read real quotes from published authors. 